\title{
A Review on Plant Derived Efflux Pump Inhibitors Targeting nor An Efflux Pump in Staphylococcus Aureus
}

\author{
Arya Mohan., Nisha A. R., V. Keerthika \\ Department of Veterinary Pharmacology and Toxicology, College of Veterinary and Animal Sciences, \\ Mannuthy, Kerala, India
}

\section{Article Info}

Volume 7, Issue 5

Page Number: 24-29

Publication Issue :

September-October-2020

\section{Article History}

Accepted : 01 Sep 2020

Published : 04 Sep 2020

\section{ABSTRACT}

Staphylococcus aureus is an important human and animal pathogen which develops resistance against various antibiotics. One of the reasons for the emergence of resistance is through efflux pumps which extrude the antibiotic out of bacterial cell. NorA is the most extensively studied efflux pumps in $S$. aureus which effluxes the fluoroquinolones, dyes and quaternary ammonium compounds. These efflux pumps can be inhibited by various natural and synthetic agents. Owing to the harmful effects of synthetic agents, the natural efflux pump inhibitors derived from various plant sources stands as a promising moiety in combating antimicrobial resistance. Even though there are many constraints in marketing the plant derived efflux pumps as therapeutic agents, it still finds a place in combating antimicrobial resistance.

Keywords : Staphylococcus aureus, NorA efflux pump, Plant derived efflux pump inhibitors

\section{INTRODUCTION}

Staphylococcus aureus is an important Gram positive organism responsible for causing a wide variety of infections ranging from superficial skin infection to life threatening septicemia in both humans as well as in animals (Fluit, 2012). The emergence of $S$. aureus as a serious pathogen is due to its ability to acquire or develop antimicrobial resistance against a wide variety of antibiotics (Floyd, 2010). Raeygaert (2018) reviewed that there are mainly four mechanisms in bacteria by which it develops antibacterial resistance. The mechanisms include limiting drug uptake by changing the cell permeability, modifying a drug target, inactivating the drug and by actively effluxing the drug. The drug efflux is the main cause for multidrug resistance (MDR) and it occurs by chromosomally coded or plasmid coded, constitutive or inducible efflux pumps present in the bacteria which have specific substrates or have a wide range of substrates for effluxing. There are five major efflux pumps in bacteria: - the ABC (ATP Binding Cassettes) superfamily, MATE (Multidrug And Toxic Extrusion) family, the SMR (Small Multidrug Resistance) family, the RND (Resistance Nodulation Division) Superfamily and the MFS (Major Facilitator Superfamily). The physiological role of efflux pump is to eliminate the noxious substances out of the cell, secretion of virulence determinants, as a stress 
responder, and efflux pumps consider the drugs as "accidental substrates" (Costa et al., 2013).

Several multidrug efflux genes are reported in S. aureus which are encoded chromosomally and plasmid encoded. The chromosome encoded efflux pumps in S. aureus include NorA, NorB, NorC, MepA, MdeA, SepA, LmrS etc. and the plasmid encoded include QacA, QacB, Smr, QacG, QacH etc (Costa et al., 2013). Among these efflux transporters the majority of the transporters belong to MFS. MFS being the largest group of solute transporter comprising of 58 families, it excludes or transports out a wide variety of substrates like amino acids, sugars, metabolites, vitamins etc. MFS are antiporters that functions as monomers in bacteria. (Floyd, 2010; Li and Nikaido, 2009).

This review tries to compile the efflux pump inhibitors that are derived from plant sources inhibiting the NorA efflux pump in $S$. aureus.

\section{Nor A Efflux Pump in S. aureus}

Costa et al. (2013) reviewed that NorA efflux pump is the most extensively studied efflux pump in $S$. aureus and the chromosomally located nor $A$ encoding gene for NorA efflux pump was isolated for the first time in a fluoroquinolone resistant strain in Japanese hospital in 1986. NorA belongs to the family MFS and uses the proton motive force for the extrusion of its various substrates like hydrophilic fluoroquinolones, quaternary ammonium compounds, various dyes like ethidium bromide, rhodamine. NorA is a protein which consists of 388 amino acids with 12 transmembrane segments. It shares similar identity with other efflux pumps like Bmr from Bacillus subtilis by $44 \%$ and with TetA which extrude tetracyclines seen in Escherichia coli by $24 \%$. Studies report that the nor $A$ gene coding for NorA efflux pump is genetically diverse with three alleles nor $A I$,
norAII, and norAIII which differ from each other upto 10 basepairs.

The resistance to fluoroquinolones, dyes or quaternary ammonium compounds mediated by NorA efflux pump can be the result of overexpression of the gene nor $A$ and this overexpression can be constitutive or inducible (Costa et al., 2013).

\section{Efflux Pump Inhibitors}

Being an important reason for mediating resistance, it is evident that inhibition of efflux pumps can potentiate the activity of antibiotics. There are many ways by which an efflux pump can be inhibited. These include: - (i) down regulation of the genes that encode the efflux pump by interfering with genetic regulation (ii) restructuring antibiotics so that they are not recognized by efflux pumps as substrates (iii) inhibit the assembly of efflux pumps (especially in RND family) (iv) blocking the efflux pump so that the substrate cannot bind to it (v) collapse the energy mechanism providing energy to the efflux pump (Pages and Amaral, 2009; Sharma et al., 2019). Efflux pump inhibitors (EPI) are molecules that inhibit the efflux pumps by any of the above mechanisms thereby preventing the drug transport out of the cell resulting in the accumulation of drugs inside the cell leading to potential activity of the drug. Efflux pump inhibitors are classified based on their origin (as plant derived EPIs, EPIs of synthetic origin and EPIs from microbes) and mechanism of action (as those inhibit energy dissipation and those inhibit direct binding) (Sharma et al., 2019).

\subsection{Plant Derived EPIs}

Medicinal plants are being used from time immemorial for treating many diseases in humans as well as in animals. These plants contain phytochemicals which are responsible for curing the 
infections. So plants can be considered as a reservoir of many phytochemicals that provide protection against many invading bacterial species (Mahmood et al., 2016). These phytochemicals can be used as an adjuvant that synergistically enhance efficacy of antibiotics (Sharma et al., 2019). Investigations are going on to find the potential of plants as source of EPIs and many plant families like Apocynaceae, Berberidaceae, Convolvulaceae, Cucurbitaceae, Fabaceae, Lamiaceae, and Zingiberaceae contain phytochemicals that are promising EPIs (Seukep et al., 2019). Stermitz et al. (2002) reported that two flavones Chysoplenol-D and Chrysoplenetin obtained from the extract of plant Artemisia annua potentiated the activity of Berberine and Norfloxacin against resistant strains of $S$. aureus by inhibiting the NorA efflux pump activity. Khan et al. (2006) showed the potentiating activity of Piperine, a major plant alkaloid in the plants Piper longum and Piper nigrum to Ciprofloxacin in reducing its MIC values against MDR strains of $S$. aureus. Through the accumulation of ethidium bromide dye inside the bacterial cells Khan et al. concluded that the potentiating activity of Piperine might be inhibiting the NorA efflux pump. Polyacylated oligosaccharide from Mexican morning glory species Orizabins are reported to strongly evoke a synergistic action with Norfloxacin against resistant $S$. aureus. The orizabins alone did not develop any antibacterial activity but shown to reverse the Norfloxacin resistance by 4 times when used at subinhibitory concentration with Norfloxacin (PeredaMiranda et al., 2006). Smith et al. (2007) through illustrating an isobologram showed an inhibitory effect on NorA function by totarol, a phenolic diterpene on the strains overexpressing NorA. Smith et al. also suggested totarol as an EPI by pointing out its efficiency in reducing the MIC of certain antibiotics. The studies of Cherigo et al. (2008) on the $\mathrm{CHCl}_{3}$ soluble plant extracts of Ipomoea murucoides yielded the pentasaccharides Murucoidins and Stoloniferins. Murucoidins potentiated the effect of
Norfloxacin by four fold and Stoloniferin by eight fold against NorA overexpressing strains of $S$. aureus at concentrations of $5-25 \mu \mathrm{g} / \mathrm{mL}$ and $5 \mu \mathrm{g} / \mathrm{mL}$ respectively. Falcao-Silva et al. (2009) reported the efflux pump inhibiting activity of Kaempferol -3-O- $\beta$ d- (6"-E-p-coumaroyl) glucopyranoside also known as tiliroside obtained from Herissantia tiubae. Tiliroside even at an MIC of $256 \mu \mathrm{g} / \mathrm{mL}$ did not show any antibacterial activity but when used alone in combination with antibiotics such as Norfloxacin, ciprofloxacin, iomefloxacin and ofloxacin reduced the MIC by 16 fold, 16 fold, four fold and two fold respectively against norA overexpressing $S$. aureus. Holler et al. (2012) reported the activity of another Kaempferol-Kaempferol-3-O-a-L-(2,4-bis-E-pcoumaroyl) rhamnoside as an EPI. This Kaempferol was obtained from the ethanolic extract of Persea lingue. This compound at a concentration of $1.56 \mathrm{mg} / \mathrm{L}$ synergistically increased the antimicrobial activity of Ciprofloxacin by eight fold against a NorA overexpressor $S$. aureus. In a study conducted by Ponnuswamy et al. (2010) Indirubin isolated from the chloroform extract of Wrightia tinctoria leaves showed a synergistic activity with Ciprofloxacin by reducing its MIC by four fold by inhibiting the Nor A efflux pump in $S$. aureus. Chan et al. (2011) determined the synergistic activity of Baicalein from Scutelleria baicalensis along with Ciprofloxacin against Nor A overexpressed strains of $S$. aureus. Kalia et al. (2012) reported the efflux pump inhibiting activity of Capsaicin obtained from hot chilli a member of genus Capsicum. Capsaicin reduced the MIC of Ciprofloxacin against a NorA overexpressing $S$. aureus as well as extending the post antibiotic effect of Ciprofloxacin by $1.1 \mathrm{~h}$ at MIC concentration. The flavonoid Sarothrin obtained from the plant Alkanna orientalis showed significant efflux pump inhibiting activity against $S$. aureus (Bame et al., 2013). Roy et al. (2013) showed the significant efflux pump inhibiting activity exhibited by coumarins isolated from Mesua ferrea against NorA overexpressed clinical strains of $S$. 
aureus. Coumarins reduced the MIC of Norfloxacin by eight fold against $\mathrm{S}$. aureus and also a dose dependent activity at sub-inhibitory concentrations. Joshi et al. (2014) while screening the antibacterial activity of thirteen phytochemicals against various efflux pumps in $S$. aureus showed significant activity against NorA efflux pump with eight and four fold reductions in MIC of Ciprofloxacin at $25 \mu \mathrm{M}$ concentrations of Osthol and Curcumin respectively. Andrade et al. (2019) showed the inhibitory activity of Brachydins obtained from Arrabidaea brachypoda against overexpressed NorA efflux pump by potentiating Norfloxacin. Ribeiro et al. (2019) reported the modulatory activity of Phyllanthin obtained from Phyllanthus amaras at sub-inhibitory concentrations towards Norfloxacin against NorA overexpressed $S$. aureus.

\section{CHALLENGES IN MARKETING PLANT DERIVED EPIs}

Since EPIs are having potentiating or synergistic activity along with antibiotics and thus aiding to overcome the antimicrobial resistance, the use of EPI in clinical therapy is far from reality. There are many constraints in developing a plant derived molecule as a therapeutic agent. The reasons can be the complex and bulk structure of the phytochemicals making them unable to synthesise in the laboratory. The other fact is that the efflux pumps are not only the mechanism in bacteria responsible for acquiring resistance. So the EPIs can overcome the resistance only to a limited extent. Since EPIs cannot be used alone and it find its use as an adjunct along with other antibiotics; the pharmacokinetics and pharmacodynamics of the EPI and the antibiotic should be similar. The EPIs are entirely a new molecule and extensive pharmcological characterisation which is important for its application in clinical therapy; of the compound is tedious, time consuming and expensive. Another important constraint is the lack of preclinical and clinical data regarding the use of EPI. The lack of information in the clinical data in animal models and supportive data on patients impedes the development of an EPI (Sharma et al., 2019; Seukep et al., 2019).

\section{CONCLUSION}

The infection with $S$. aureus is increasing alarmingly and so the antimicrobial resistance. Antimicrobial resistance stands a big threat in treating the infections caused by the organisms. One of the main reasons for emergence of multidrug resistant strains of $\mathrm{S}$. aureus is the overexpression of the efflux pumps which extrude the antibiotic molecules from the cell into the environment. The use of EPIs that can block or inhibit the efflux pumps can prevent the resistance mediated by efflux pumps. Owing to the adverse effect produced by the chemical or synthetic EPIs in mammalian body, the use of such agents can be avoided or minimized using novel harmless agents like EPIs derived from plant sources. Even though they are not available in the market, the potential of plant derived EPIs are promising strategies in combating the antibiotic resistance in plants.

\section{FUTURE PERSPECTIVES}

The aim should be to make the plant derived EPIs available in the market for clinical therapy. The constraints in the development of the product should be investigated and eliminated. Researches should focus on making ample data on preclinical and clinical data on the use of EPIs in both animal model sand human patients.

\section{REFERENCES}

[1]. Bame, J.R., Graf, T.N., Junio, H.A., Bussey III, R.O., Jarmusch, S.A., El-Elimat, T., Falkinham III, J.O., Oberlies, N.H., Cech, R.A. and Cech, 
N.B. 2013. Sarothrin from Alkanna orientalis is an antimicrobial agent and efflux pump inhibitor. Planta. Med. 79: 327-329.

[2]. Chan, B.C., Ip, M., Lau, C.B., Lui, S.L., Jolivalt, C., Ganem-Elbaz, C., Litaudon, M., Reiner, N.E., Gong, H., See, R.H. and Fung, K.P. 2011. Synergistic effects of baicalein with ciprofloxacin against NorA over-expressed methicillinresistant Staphylococcus aureus (MRSA) and inhibition of MRSA pyruvate kinase. J. Ethnopharmacol. 137: 767-773.

[3]. Cherigo, L., Pereda-Miranda, R., FragosoSerrano, M., Jacobo-Herrera, N., Kaatz, G.W. and Gibbons, S. 2008. Inhibitors of bacterial multidrug efflux pumps from the resin glycosides of Ipomoea murucoides. J. Nat. Prod. 71: 10371045.

[4]. Costa, S.S., Viveiros, M., Amaral, L. and Couto, I. 2013. Multidrug efflux pumps in Staphylococcus aureus: an update. Open Microbiol. J. 7: 59-71.

[5]. De Sousa Andrade, L.M., de Oliveira, A.B.M., Leal, A.L.A.B., de Alcantara Oliveira, F.A., Portela, A.L., Neto, J.D.S.L., de Siqueira-Junior, J.P., Kaatz, G.W., da Rocha, C.Q. and Barreto, H.M. 2020. Antimicrobial activity and inhibition of the NorA efflux pump of Staphylococcus aureus by extract and isolated compounds from Arrabidaea brachypoda. Microb. Pathog.140. https://doi.org/10.1016/j.micpath.2019.103935

[6]. Falcao Silva, V.S., Silva, D.A., Souza, M.D.F.V. and Siqueira Junior, J.P. 2009. Modulation of drug resistance in Staphylococcus aureus by a kaempferol glycoside from Herissantia tiubae (Malvaceae). Phytother. Res. Int. J. Pharmacol. Toxicol. Eval. Nat. Prod. Deriv. 23: 1367-1370.

[7]. Floyd, J.L., Smith, K.P., Kumar, S.H., Floyd, J.T. and Varela, M.F. 2010. LmrS is a multidrug efflux pump of the major facilitator superfamily from Staphylococcus aureus. Antimicrob. Agents Chemother. 54: 5406-5412.
[8]. Fluit, A.C. 2012. Livestock-associated Staphylococcus aureus. Clin. Microbiol. Infect. 18: 735-744.

[9]. Holler, J.G., Christensen, S.B., Slotved, H.C., Rasmussen, H.B., Gúzman, A., Olsen, C.E., Petersen, B. and Molgaard, P. 2012. Novel inhibitory activity of the Staphylococcus aureus NorA efflux pump by a kaempferol rhamnoside isolated from Persea lingue Nees. J. Antimicrob. Chemother. 67: 1138-1144.

[10].Joshi, P., Singh, S., Wani, A., Sharma, S., Jain, S.K., Singh, B., Gupta, B.D., Satti, N.K., Koul, S., Khan, I.A. and Kumar, A. 2014. Osthol and curcumin as inhibitors of human Pgp and multidrug efflux pumps of Staphylococcus aureus: reversing the resistance against frontline antibacterial drugs. Med. Chem. Comm. 5: 15401547.

[11].Kalia, N.P., Mahajan, P., Mehra, R., Nargotra, A., Sharma, J.P., Koul, S. and Khan, I.A. 2012. Capsaicin, a novel inhibitor of the NorA efflux pump, reduces the intracellular invasion of Staphylococcus aureus. J. Antimicrob. Chemother. 67: 2401-2408.

[12].Khan, I.A., Mirza, Z.M., Kumar, A., Verma, V. and Qazi, G.N. 2006. Piperine, a phytochemical potentiator of ciprofloxacin against Staphylococcus aureus. Antimicrob. Agents Chemother. 50: 810-812.

[13].Li, X.Z. and Nikaido, H. 2009. Efflux-mediated drug resistance in bacteria. Drugs. 69: 1555-1623.

[14].Mahmood, Y.H., Jamshidi, S., Mark Sutton, J. and M Rahman, K. 2016. Current advances in developing inhibitors of bacterial multidrug efflux pumps. Curr. Med. Chem. 23: 1062-1081.

[15].Pages, J.M. and Amaral, L. 2009. Mechanisms of drug efflux and strategies to combat them: challenging the efflux pump of Gram-negative bacteria. Biochim. Biophys. Acta Proteins Proteom. 1794: 826-833. 
[16].Pereda-Miranda, R., Kaatz, G.W. and Gibbons, S. 2006. Polyacylated oligosaccharides from medicinal Mexican morning glory species as antibacterials and inhibitors of multidrug resistance in Staphylococcus aureus. J. Nat.Prod. 69: 406-409.

[17].Ponnusamy, K., Ramasamy, M., Savarimuthu, I. and Paulraj, M.G. 2010. Indirubin potentiates ciprofloxacin activity in the NorA efflux pump of Staphylococcus aureus. Scand. J. Infect. Dis. 42: 500-505.

[18].Reygaert, W.C. 2018. An overview of the antimicrobial resistance mechanisms of bacteria. AIMS microbiol. 4: 482-501.

[19].Ribeiro, A.M.B., de Sousa, J.N., Costa, L.M., de Alcântara Oliveira, F.A., dos Santos, R.C., Nunes, A.S.S., da Silva, W.O., Cordeiro, P.J.M., Neto, J.D.S.L., de Siqueira-Júnior, J.P. and Kaatz, G.W. 2019. Antimicrobial activity of Phyllanthus amarus Schumach. \& Thonn and inhibition of the NorA efflux pump of Staphylococcus aureus by Phyllanthin. Microb. Pathog. 130: 242-246.

[20].Roy, S.K., Kumari, N., Pahwa, S., Agrahari, U.C., Bhutani, K.K., Jachak, S.M. and Nandanwar, H. 2013. NorA efflux pump inhibitory activity of coumarins from Mesua ferrea. Fitoterapia. 90: 140-150.

[21].Seukep, A.J., Kuete, V., Nahar, L., Sarker, S.D. and Guo, M. 2019. Plant-derived secondary metabolites as the main source of efflux pump inhibitors and methods for identification. J. Pharm. Anal. Available: https://doi.org/10.1016/j.jpha.2019.11.002

[22].Sharma, A., Gupta, V.K. and Pathania, R. 2019. Efflux pump inhibitors for bacterial pathogens: From bench to bedside. Indian J. Med. Res. 149: 129-145.

[23].Smith, E.C., Kaatz, G.W., Seo, S.M., Wareham, N., Williamson, E.M. and Gibbons, S. 2007. The phenolic diterpene totarol inhibits multidrug efflux pump activity in Staphylococcus aureus. Antimicrob. Agents Chemother. 51: 4480-4483.

[24].Stermitz, F.R., Scriven, L.N., Tegos, G. and Lewis, K. 2002. Two flavonols from Artemisa annua which potentiate the activity of berberine and norfloxacin against a resistant strain of Staphylococcus aureus. Planta Med. 68:11401141.

\section{Cite this article as :}

Arya Mohan., Nisha A. R., V. Keerthika, "A Review on Plant Derived Efflux Pump Inhibitors Targeting nor An Efflux Pump in Staphylococcus Aureus", International Journal of Scientific Research in Science and Technology (IJSRST), Online ISSN : 2395-602X, Print ISSN : 2395-6011, Volume 7 Issue 5, pp. 24-29, September-October 2020. Available at doi : https://doi.org/10.32628/IJSRST20754

Journal URL : http://ijsrst.com/IJSRST20754 